\title{
Silent sufferers: Health care practitioners as second victims of patient safety incidents
}

\author{
Brenda Kubheka ${ }^{1 *}$, Shan Naidoo ${ }^{1}$, Edwin Etieyibo ${ }^{2}$ and Khulekani Moyo ${ }^{3}$ \\ ${ }^{1}$ School of Public Health, University of Witwatersrand, South Africa \\ ${ }^{2}$ School of Philosophy, University of Witwatersrand, South Africa \\ ${ }^{3}$ School of Law, University of the Witwatersrand, South Africa
}

\section{Introduction}

Patient safety is defined as the avoidance, prevention and amelioration of adverse outcomes or injuries or harm to patients [1]. A patient safety incident (PSI) is caused by the management rather than the disease process and is sufficiently serious to lead to prolongation of hospitalisation or to temporary or permanent impairment or disability to the patient at the time of discharge or both and even death [1,2]. It is important to recognise that most practitioners care about their patients and their professional reputations hence the inference that majority of these incidents are unintended and do not reflect the carelessness of health care practitioners (HCP). Importantly, these incidents tend to be multisectorial $[3,4]$. Of interest, pregnant women attended by obstetrician expect a perfect delivery and a live baby. Regrettably, medicine is an imperfect science [5] and humans are fallible.

PSIs have a direct impact on the wellbeing of HCPs and indirect impact on their performance. There is evidence that PSIs affect HCPs professionally, emotionally, and socially thus qualifying them as 'second victims', a term coined by $\mathrm{Wu}[4,6-8]$. Second victims are defined as "health practitioners who are involved in an unanticipated adverse patient event, in a medical error and/or a patient related injury and become victimized in the sense that the provider is traumatized by the event. Frequently, these individuals feel personally responsible for the patient outcome. Many feel as though they have failed the patient, second guessing their clinical skills and knowledge base" [6,9]. Medical errors cause significant harm to patients and alarmingly, one report suggesting them as the third leading cause of death in the USA $[8,10]$. Similar data is not available for the South African context. Relevant to this paper is the study conducted by Wilson, et al. assessing the nature and scale of patient harm in developing countries, including South Africa (SA). This study involved 26 hospitals chosen from 8 countries and cites $8.2 \%$ of the reviewed medical records showed at least one adverse event, with a range of $2.5 \%$ to $18.4 \%$ per country. Interestingly, $85 \%$ of these incidents were deemed preventable and approximately $30 \%$ were associated with death [11]. Therefore, patient safety qualifies as a public health concern and a human rights issue [1].

The standard response to PSIs by health establishments is firstly, to attend to patients and relatives followed by addressing organizational issues, like notifying the legal team. HCPs are the least attended stakeholders [8]. Patients and their families are considered first victims of PSIs, HCPs as second victims and the health establishment as a third victim [10]. Medicine, is an imperfect science and not all adverse outcomes are a result of medical errors or negligence [5,10]. For the purpose of this paper, PSIs will refer to all harm causing adverse events, which may lead to prolonged hospitalisation, temporal or permanent disability or death of the patient, fetus or baby. This paper seeks to explore the interplay between the health system, patient safety culture, and second victimhood.

\section{Discussion}

\section{The health system}

The health system is manned by autonomous HCPs and it accepts deviation from protocols and standard guidelines a long as the instructions come from seniors. This is different from other risk prone industries, for example aviation, where errors and expertise are seen as a by-product of a system and an individual [9]. The deviations and poor design of the health system creates many opportunities for errors to happen. The Swiss cheese model of human errors attributes medical errors to the combination active errors and latent conditions from the weaknesses in the regulatory and management domains [12].

The multi-country study conducted in developing countries identified the following as contributing factors to harmful incidents (1) Inadequate training or supervision of clinical staff, (2) No protocol, policy or failure to implement, (3) Inadequate communication or reporting, (4) Delay in providing service, (5) Defective equipment or supplies, (6) Unavailable equipment and supplies, (7) Inadequate functioning of hospital services and, (8) Inadequate staffing [11]. This raises questions about both legal and moral duty employers and managers of HCPs have towards second victims in such environments. HCPs also get frustrated when attending to emergencies and get confronted with inadequate resources and failing procedures. This get worsened when a person with no clinical responsibility is responsible for allocation and provision of clinical resources. Compounding this challenge is the 'normalization of deviance', which is a product of desensitization to circumstances and practices that are risky and including those considered an inconvenience to patients. These circumstances and practices ought to be deemed unacceptable in health care [12].

To improve safety, the dynamic nature of the health system ought to be embraced and this includes acknowledgement that changes in the

${ }^{\star}$ Correspondence to: Brenda Kubheka, School of Public Health, University of Witwatersrand, South Africa, E-mail: brenda.k@wol.co.za

Received: November 08, 2019; Accepted: November 18, 2019; Published: November 21, 2019 
system may erode or enhance safety [12]. Vincent, Burnett and Carthey developed the patient safety measurement framework which comprises of five elements; (1) Past harm which looks at the past physical and psychological harm on patients, (2) Reliability which looks at the reliability of the system and procedure in the health system and the capability of people to uphold these, (3) Sensitivity to operations which looks at the continuous monitoring of safety, (4) Anticipation and preparedness which looks at the anticipation and preparedness for safety in the future and, (5) Integration and learning which looks at the ability of the organisation to respond, learn and utilize the information. The model facilitates reflection and identification of gaps by HCPs and health establishments [13-18].

\section{Safety culture, perfectionism and attitude towards PSIs}

The medical profession is fraught with a culture of perfectionism that fails to prepare professionals for dealing with medical errors [5]. It does seem that this culture and attitude have an impact on the management of PSIs in an inauspicious manner thus casting emotional burden on HCPs [14]. A culture of perfectionism and infallibility seems to form part of medical training and this encourages internalization and even hiding of errors creating a fertile ground for emotional and moral burden. Disclosure of errors and maintaining good relations with patients following PSIs is associated with better emotional outcomes for professionals although they sometimes get discouraged to disclose errors by their seniors and legal practitioners [11]. Disclosure allows the health system to appreciate its weaknesses and the responsibility to implement continuous improvement initiatives. Vitally, disclosure seeks to restore trust and maintain the therapeutic relationship between HCP and the patient.

Vincent asserts that it is unreasonable to expect HCPs to be open about incidents if they anticipate negative sanctions from their seniors. Fear of litigation and media attention intensifies the fear experienced by HCPs involved in major PSIs, notwithstanding the additional burden imposed by complaints and litigations [17]. Worryingly, is the willingness of HCPs to ask for help being influenced by the level of competitiveness amongst colleagues. Some HCPs worry about being perceived as weak when they ask for help [9]. A competitive and unsupportive surgical culture observed amongst surgeons discourages HCPs to shout for help [4].There is no evidence supporting the existence or lack of a similar culture amongst HCPs working in other specialties.

Some HCPs have experienced aggression, blame, interrogation, and even threats following PSIs $[11,16]$. It is not surprising that this kind of response to PSI enables poor self-reporting of errors. A professional community that acknowledges its error-proneness, is more likely to put safety measures in place to prevent errors from happening and also support those involved in errors [9]. Poor communication, lack of empathy and failure to provide support following PSIs causes secondary harm to HCPs. Evidence suggests that communication with peers and seniors are perceived as very helpful although lacking. Interestingly, lack of support is perceived to be linked to the personality of the HCP involved in the error $[12,19]$. A just patient safety culture is vital and it ought to be grounded on continuous improvement and learning from errors [8]. This safety culture is defined by five interrelated attributes, which are being an informed culture, a reporting culture, a just culture, a flexible culture and a learning culture. It avoids blame at the same time ensuring accountability in cases of negligent care [19].

\section{Emotional response to PSIs}

A study conducted in a Boston based academic hospital cited $84 \%$ of surgeons experiencing significant emotional and professional toll following intraoperative adverse event $[4,5]$. The emotional response comprised of a combination of anxiety, guilt, anger, sadness, shame, blaming others, and loss of confidence for months or years. Professionals also acknowledged the concern they have about their reputations, competence and even question their career choices following these events $[4,5,9,11]$. Majority of these surgeons valued and sought support from their peers and to the contrary, the peers willingness to support second victims is lacking. In addition, HCPs also suffer in silence because of the risk of being perceived by others as being incompetent and the fear of poor management of PSIs by establishments $[4,11]$.

The emotional response to preventable harm evokes emotional and psychological responses and this is termed Second Victim Syndrome (SVS). It is estimated that nearly $50 \%$ of HCPs have experienced SVS in their career and the likelihood increases with burnout. Burnout is characterized by emotional exhaustion and depersonalization. This is linked to increased patient dissatisfaction, decreased quality of care and an increased likelihood of PSIs thus creating a vicious cycle (Figure 1). HCP wellbeing, resilience and their ability to manage stress are important factors in patient safety management $[8,16,19,20]$.

According to Scott, et al. all study participants regardless of gender, professional background or years of experience easily recalled the immediate and ongoing impact of PSIs [5]. This is contrary to another study citing $79 \%$ of surgeons involved in PSIs with 10 or more years of experience as surgeons, did not experience any negative emotions following an intraoperative adverse events. Juxtaposed to this finding is a comment by one of the surgeons stating that they hide their grief and suffer in silence [2]. Critically, HCPs cited second victimhood as a lifealtering experience leaving a permanent imprint in a person's life [16]. Acknowledgement of the difference in temperament and resilience of HCPs is vital and its influence on responses to their mistakes. HCPs with high personal standards and that are also highly self-critical are more likely to suffer emotional consequences of errors [17]. There is a study conducted amongst midwives and obstetrician that cited a fifth of these HCPs involved in PSIs were no longer involved in the delivery care and $25 \%$ of them had resigned due to the burden of responsibility [21]. Some HCPs may find dysfunctional ways of dealing with the emotional burden and these include the use of alcohol and drugs [22].

\section{Organisational support following PSIs}

It is estimated that nearly half of HCPs will experience the impact of being a second victim at least once in their career [22]. Vitally, interventions and support for second victims ought to be directed at offering 'emotional first aid' to HCPs and this ought to include excusing

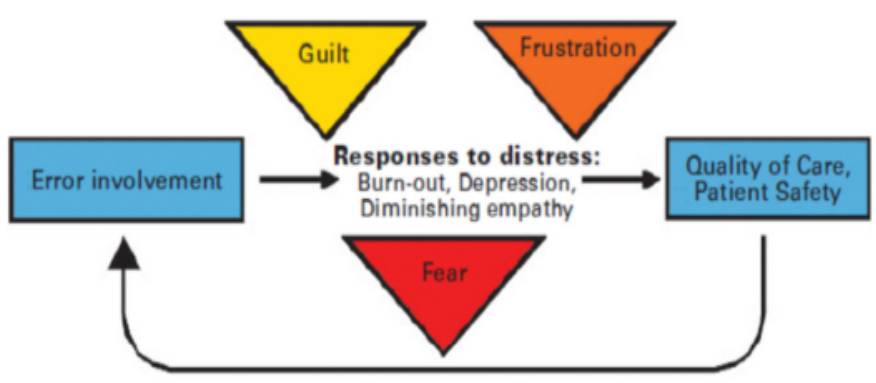

Figure 1. Cycle of error, burnout and error. (Source: Schwappach and Boluarte, 2008) 
them from normal duties coupled with emotional support from peers, seniors professional counselors or, the clergy. This approach will benefit first, second and third victims [8] thus reducing negative impact on the entire health system. There is no doubt that establishments ought to provide support to HCPs involved in preventable PSIs and any other unexpected outcome. Of concern, is a study citing $68 \%$ of HCPs involved in these incidents did not receive any institutional support [5].

The challenge of lacking support is compounded by the absence of programs for supporting affected HCPs although it is thought that establishments have untapped resources to cater for this need $[5,8]$. Poor support may lead to HCPs addressing their negative emotions independently, which may lead to self-defense, depersonalization of the incident and even suicidal thoughts $[4,16]$. A study conducted on anaesthesiologists cites that only $7 \%$ of involved in major incidents were given a break from their duties following a major PSI. Most respondents cited logistical issues related unavailability of resource capacity to take over their workload as the major barrier to being relieved from work. This is unfortunate because most professionals had to go back to work immediately after the incident [16]. Lack of support and inability to deal with medical errors might lead to dropout of professionals and this may include quitting the profession, changing roles to the ones with less patient contact, or move to a new unit because of the negative impact of PSIs on their quality of life $[5,8]$. Unfortunately PSIs sometime get attributed to poor character trait thus making errors a source of shame and therefore not easy to report [9].

Some of the emotional distress is exacerbated by the workplace culture that enables gossiping and self-doubt $[6,16]$. Supporting second victims is a moral imperative and it is good medicine [16]. There is a tendency of treating Mortality and Morbidity (M\&M) meetings as a tracking tool for incidents and sometimes undermining the importance of creating a database of all incidents and learning from all PSIs. M\&M meetings are perceived to be accusatory, threatening, sometimes hostile and focused on blaming than addressing safety issues. These meetings also fail to offer emotional support to HCPs involved in PSIs [4,8]. Disturbingly, is a study conducted on defensive medical practice amongst South African neurosurgeons citing that $76 \%$ of the respondents refer patients to other HCPs to reduce their own risk to litigation and $89 \%$ of respondents confirmed requesting imaging studies that are not clinically indicated but do so for the purpose of minimizing litigation exposure. The same study cited almost that $60 \%$ of respondents would not choose post-graduate studies in neurosurgery if they were to start all over again [23-26]. This is worrying because a similar attitude may filter to the trainees and defensive medical practice drives health care costs.

\section{The role of ethics in patient safety and second victimhood}

An ultimate patient-HCP relationship is the one characterized by cooperation, trust and shared decision-making. It ought to be groundedon full disclosure of medical errors as an enabler of protecting this relationship against harm [23,24]. The fiduciary relationship between the patient and the HCP ought to be based on trust, justice, non-maleficence and beneficence [25]. Disclosure of errors has a positive impact on the wellbeing of HCPs, preserves the patient-HCP relationship, and it facilitates continuation of care. It is cited that disclosure may cause additional stress [21] and this is not surprising because of the breach of the duty to care leading to unintended harm. Compounding this, is the fear of disclosed information being used against HCPs.
There is a glaring need for health leaders and service managers to establish formal and informal programs to support second victims [19]. Of concern, is the debate around the legal recognition of second victimhood and debates about the term itself. Managers have a moral duty to establish these programs to support the harmed patients and their families. Supporting HCPs protects other patients from harm. Undoubtedly, managers ought to investigate PSIs and contributing factors to facilitate quality improvement initiatives and prevent recurrances [22]. Immediate and long-term needs of affected practitioners ought to be taken into account and programs ought to be aligned to these needs [22]. Health service managers ought to take heed of five rights of the second victims identified by Denham, using the acronym TRUST and these are (1) Treatment that is just, (2) Respect, (3) Understanding and compassion, (4) Supportive care, and (5) Transparency and the opportunity to contribute to learning [7]. According to Seys and Wu, et al. "HCPs involved in medical errors get hurt too. If we are healers, then we have a job of healing them too. This is not an elective but an ethical issue" [22].

\section{Conclusion}

The objective of healthcare is to promote wellness, save lives, heal, and improve quality of life. HCPs do get impacted negatively both emotionally and professionally when these objectives get compromised unintentionally during the delivery of care. Second victimhood has negative impact on patient safety and the professionals' wellbeing therefore making it an ethical and a human rights issue. Health service managers ought to acknowledge the relationship between safety culture, management of PSIs and their negative impact on HCPs.

\section{Recommendations}

Concerted efforts must be made to improve awareness of second victimhood amongst trainees, HCPs and health service managers. Support programs with clear referral mechanisms must be developed to support HCPs involved in PSIs. Studies have shown that HCPs want support from their peers and seniors therefore, these stakeholders ought to be trained on how to support second victims in the workplace.

\section{Acknowledgements}

The authors would like to thank Albert Wu and Charles Vincent for their valuable inputs.

\section{References}

1. Kohn L, Corrigan J, Donaldson M (2000) To err is human: Building a safer health system: National Academy Press, Washington (DC), (US).

2. Vincent C (2011) Essentials of Patient Safety: Willey-Blackwell, London.

3. Merry AF (2009) How Does the Law Recognise and Deal with Medical Errors? In Hurwitz B, Sheikh A (Eds), In Health Care Errors and Patient Safety.

4. Han K, Bohnen JD, Peponis T, Martinez M, Nandan A, et al. (2017) The Surgeon as the Second Victim? Results of the Boston Intraoperative Adverse Events Surgeons' Attitude (BISA) Study. J Am Coll Surg 224: 1048-1056. [Crossref]

5. Adinma J (2016) Litigations and the Obstetrician in Clinical Practice. Ann Med Health Sci Res 6: 74-79. [Crossref]

6. Wu Albert W (2000) Medical error: the second victim. The doctor who makes the mistake needs help too. BMJ 320: 726. [Crossref]

7. Scott SD, Hirschinger LE, Cox KR, McCoig M, Brandt J, et al. (2009) The natural history of recovery for the healthcare provider, 'second victim', after adverse patient events. Qual Saf Health Care 18: 325-330. [Crossref]

8. Marmon LM, Heiss K (2015) Seminars in Pediatric Surgery Improving surgeon wellness: The second victim syndrome and quality of care. Semin Pediat Surg 24: 315-318. 
9. Santomauro CM, Kalkman CJ, Dekker SWA (2014) Second victims, organizational resilience and the role of hospital administration. Journal of hospital administration 3: 95-103.

10. Coughlan B, Powell D, Higgins MF (2017) The Second Victim: A Review. Eur J Obstet Gynecol Reprod Biol 213: 11-16. [Crossref]

11. Wilson RM, Michel P, Olsen S, Gibberd RW, Vincent C, et al. (2011) Patient safety in developing countries: retrospective estimation of scale and nature of harm to patients in hospital. $B M J$ 344: e832.

12. Runciman B, Merry A, Walton M (2007) Safety and ethics in healthcare: A guide to getting it right. CRC Press, London.

13. Carthey J (2013) Understanding safety in healthcare: The system evolution, erosion and enhancement model. J Public Health Res 2: 144 -149. [Crossref]

14. Sirriyeh R, Lawton R, Gardner P, Armitage G (2010) Coping with medical error: a systematic review of papers to assess the effects of involvement in medical errors on healthcare professionals' psychological well-being. BMJ Qual Saf 19: 1-8.

15. Bynum WE, Goodie JL (2014) Shame, guilt, and the medical learner: Ignored connections and why we should care. Med Educ 48: 1045-1054.

16. Pratt SD, Jachna BR (2015) Care of the clinician after an adverse event. Int J Obstet Anesth 24: 54-63. [Crossref]

17. Vincent C (2003) Understanding and Responding to Adverse Events. $N$ Engl J Med 348: 1051-1056
18. Vincent C, Burnett S, Carthey J (2014) Safety measurement and monitoring in healthcare: a framework to guide clinical teams and healthcare organisations in maintaining safety. BMJ Qual Saf 23: 670-677. [Crossref]

19. Reason J (1998) Achieving a safe culture: Theory and practice. Work \& Stress 12: 293 306

20. Schwappach DL, Boluarte TA (2008) The emotional impact of medical error involvement on physician. Swiss Med Wkly 138: 9-15.

21. Wahlberg $\AA$, Andreen Sachs M, Johannesson K, Hallberg G, Jonsson M, et al. (2017) Post-traumatic stress symptoms in Swedish obstetricians and midwives after severe obstetric events: a cross-sectional retrospective survey. BJOG 124: 1264-1271.

22. Seys D, Wu AW, van Gerven E, Vleugels A, Euwema M, et al. (2012) Health Care Professionals as Second Victims after Adverse Events: A Systematic Review. Eval Health Prof 36: 135-162. [Crossref]

23. Beauchamp TL, Childress JF (2013) Principles of Biomedical Ethics: Oxford University Press, New York.

24. Allan A, Mckillop D (2010) The health implications of apologizing after an adverse event. Int J Qual Health C 22: 126-131.

25. Chamberlain CJ, Leonidas BA, Wu AW, Pawlik TM (2017) Disclosure of "Nonharmful" Medical Errors and Other Events. Arch Surg 147: 282-286.

26. Roytowski D, Smith TR, Fieggen AG, Taylor A (2014) Impressions of defensive medical practice and medical litigation among South African neurosurgeons. S Afr Med $J$ 104: 736-738. [Crossref]

Copyright: (C2019 Kubheka B. This is an open-access article distributed under the terms of the Creative Commons Attribution License, which permits unrestricted use, distribution, and reproduction in any medium, provided the original author and source are credited. 